\title{
Differential eyelid conditioning: The generalization of reinforcement and of nonreinforcement*
}

\author{
FREDERICK L. NEWMAN $\dagger$ \\ University of Pennsylvania Medical School \\ Philadelphia, Pa. 19104 \\ and \\ JAMES C. FRANCIS, ALICE WEST
and DIANE COVEY
University of Miami, Coral Gables, Fla. 33124
}

In two human differential eyelid studies, Ss were first differentially conditioned to two tonal CSs, after which a third $\mathrm{CS}\left(\mathrm{CS}_{3}\right)$ was introduced in random sequence with the established $\mathrm{CS}+$ and $\mathrm{CS}-$. The quality of $\mathrm{CS}_{3}$ was either a red light (Experiment I) or a tone (Experiment II). As a tone (Experiment II), $\mathrm{CS}_{3}$ was either more similar to $\mathrm{CS}+$ than $\mathrm{CS}-$, or vice versa. In both studies, the percent of $\mathrm{CS}_{3}$-UCS pairing varied factorially between groups as $0 \%, 20 \%$, or $60 \%$ of the $\mathrm{CS}_{3}$ trials. As was true in earlier studies, the historic notions of reinforcement and stimulus generalization were not adequate to explain the results.

The two experiments were designed to estimate the effects generalized from established CS+ and CS- by introducing a third $\mathrm{CS}, \mathrm{CS}_{3}$, into random sequence with $\mathrm{CS}+$ and CS- after differential responding was obtained.

\section{EXPERIMENT I}

The first experiment was designed to demonstrate the effects of introducing a new association ( $\mathrm{CS}_{3}$-UCS) in addition to the associations already established in the initial differential conditioning phase. To eliminate differential generalization from either CS+ or CS- to $\mathrm{CS}_{3}, \mathrm{CS}_{3}$ differed in modality from CS+ and CS-. By varying the percent of $\mathrm{CS}_{3}$-UCS pairings (between groups) the design allowed for an estimate of the effect of introcuding a new association, where the strength of $\mathrm{CS}_{3}$-UCS association varied with percent $\mathrm{CS}_{3}$-UCS pairing.

\section{Method}

\section{Apparatus}

An $\mathrm{S}$ sat facing the back wall of a $1.219 \times 1.523 \mathrm{~m}$ enclosure, painted flat white, an 8-ohm speaker centered in the ceiling, and an IEE display cell encased in a $152 \times 152 \times 304 \mathrm{~mm}$ box at eye level on the back wall. The $S$ was fitted with a Waltke headband, which supported an airjet (1.53-mm orifice) and a Gianninni Minitorque rotary potentiometer (Model 35153). The latter was connected by tape and light piano wire to the S's upper right

*The research was supported in part by an NIH institutional grant, University of Miami. The two experiments reported here were presented at the 1970 and 1971 meetings of the Psychonomic Society. The paper was largly prepared while the first author was a Fellow at the Center for Advanced Studies in the Behavioral Sciences, Palo Alto, California. The authors would like to acknowledge the assistance of Susan Anderson and John Rost. Paritcularly, acknowledgment should be made of Juan Pino's long hours of labor on this project.

tRequests for reprints should be sent to Frederick L. Newman, EPPI, Henry Avenue and Abbottsford Road, Philadelphia, Pa. 19129 eyelid. The resistance changes relayed from the potentiometer were amplified and recorded on an Offner Type $R$ recorder.

All CSs had a 900-msec duration, and on reinforced trials a CS terminated with a $100-\mathrm{msec}$ duration puff of nitrogen, providing an $800-\mathrm{msec}$ CS-UCS interval. The UCS intensity was $125 \mathrm{~mm} \mathrm{Hg}$ static pressure $(2.45 \mathrm{psi})$. Four intertrial interval values of $9,12,15$, and $18 \mathrm{sec}$ (13.5 sec average) were randomized. The two tonal CSs were hertz values of 600 and 1,000 at $75 \mathrm{~dB}$ SPL, generated by Hewlett-Packard oscillators (Model 204D). The assignment of the 600 - or the $1,000-\mathrm{Hz}$ tones as CS+ and CS - was counterbalanced among Ss. The third CS $\left(\mathrm{CS}_{3}\right)$ was a red light emitted from a $28-\mathrm{V}$ dc bulb in the IEE display cell. A Grason-Stadler (Model 901-B) noise generator, at $70 \mathrm{~dB}$ SPL intensity, masked extraneous sounds.

\section{Procedure and Design}

Following "neutral" instructions, each S received three blocks of 24 differential conditioning trials involving the random sequencing of $12 \mathrm{CS}+$ and $12 \mathrm{CS}$ - trials per trial block. Following the initial differential conditioning phase, each $S$ experienced two blocks of 60 trials with the three CSs presented in random sequence 20 times per block. Three groups of Ss were distinguished by the percent of total $\mathrm{CS}_{3}$ trials, which were reinforced (paired) with the UCS, $0 \%, 20 \%$, or $60 \%$. The design involved two levels of counterbalancing (of the two hertz values of tones as CS+ and CS-) and three values of percent $\mathrm{CS}_{3}-\mathrm{UCS}$ pairings. The three levels of percent $\mathrm{CS}_{3}$-UCS were selected on the basis of previous work (Peterson \& Newman, 1970).

The criterion of reliable differentiation during the first training phase was derived from a post hoc analysis of a separate pilot study. The 12 male pilot Ss received 96 differential conditioning trials. The pilot data showed that for 9 of the 12 Ss, if a difference score of $41.67 \%$ more CRs to CS+ was obtained on Trials 49-72, then a difference score of at least $25 \%$ was observed over Trials 73-96. For the actual experiment, each $S$ was assigned randomly to one of the six groups and run accordingly. If the S's difference score on Trials 49-72 was at least $41.67 \%$, he was then labeled as a "good" (i.e., reliable) differentiating $S$. When a particular cell of the 2 by 3 design had six "good" Ss, assignment of Ss to that cell was discontinued.

\section{Subjects}

Eighty-five male introductory psychology students were run. ${ }^{1}$ Data for six Ss were discarded because of apparatus failure and experimental error. There were $81 \%$ of the Ss showing positive difference scores. By the criterion of the $41.67 \%$ difference score on Trials 49-72, however, $36 / 79$ Ss were "good" differentiating Ss; $28 / 79$ showed positive difference scores below the criterion $41.67 \%$ ("poor" differentiators). Using the overall observed ratios of good, poor, and nondifferentiating Ss to estimate the expected number of each kind of differentiator in each of the six partitions, no significant departures from the expected frequencies were found (all $\mathrm{p}$ values $>.10$ ).

\section{Results and Discussion}

The results of only the reliable ("good") differentiators were analyzed in full. ${ }^{2}$ The means of poor and nondifferentiating Ss showed response patterns similar to the "good" differentiators; however, problems of empty and unbalanced cells and meeting distribution assumptions precluded analysis of the poor and nondifferentiating Ss' data. The two panels of Fig. 1 represent the percent CRs to each of the three CSs 


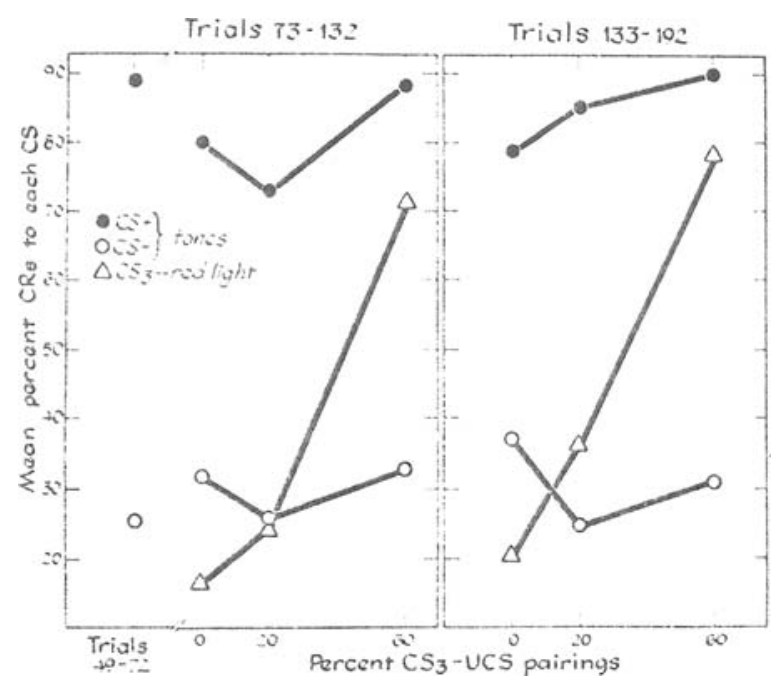

Fig. 1. The mean percent CRs to each CS for Experiment I are presented. The left-hand panel gives the percent CRs to CS+, $\mathrm{CS}-$, and $\mathrm{CS}_{3}$ by percent $\mathrm{CS}_{3}$-UCS pairing average over Trials 73-132. The first point on the abscissa of the left-hand panel represents the last block of CS+ and CS- trials prior to the introduction of $\mathrm{CS}_{3}$ (Trials 49-72). The right-hand panel provides the mean percent CRs to each CS for Trials 133-192.

during the last two blocks of 60 trials. The left panel also presents the percent CRs to CS+ (closed circle) and CS- (open circle) for the trial block preceding the introduction of $\mathrm{CS}_{3}$ (Trials 49-72). The percent CRs to $\mathrm{CS}+$ and CS- on Trials 49.72 provide reference points representing the levels of responding prior to introducing $\mathrm{CS}_{3}$ trials.

As the percent $\mathrm{CS}_{3}$-UCS pairings increased, there was a significant linear increase in percent $\mathrm{CRs}$ to $\mathrm{CS}_{3}$, $F(1,30)=40.45, p<.001$. The $\mathrm{CS}_{3}$ results were anticipated and represent the typical partial reinforcement acquisition effects.

Using the percent CRs to CS+ on Trials $49-72$ as a reference point, the percent CRs to CS+ were contrasted for each of three groups. When percent $\mathrm{CS}_{3}$-UCS pairing was $0 \%$ or $20 \%$, the percent CRs over CS+ trials showed a decrease on the first block of 60 trials after $\mathrm{CS}_{3}$ introduction (Trials 73-132), $\mathrm{F}(1,30)=15.83, \mathrm{p}<.01$. On the other hand, only the $0 \%$ group (and not the $20 \%$ group) retained the depressed level of percent CRs to CS+ on the last block of trials, $F(1,30)=4.81, p<.05$. In summary, then, there was a significant and lasting decrease in response tendency to $\mathrm{CS}+$ when percent $\mathrm{CS}_{3}$-UCS pairing was zero, while only temporary decrease was observed when percent $\mathrm{CS}_{3}$-UCS pairing was low $(20 \%)$ and no depression of response tendency to $\mathrm{CS}+$ was observed when percent $\mathrm{CS}_{3}$-UCS was high (60\%). Assuming that the percent CRs to CS+ in the $60 \%$ group were at a ceiling of responding, then the results would be expected on the basis of an overall increase in the number of reinforcements per 60 -trial block.

Initially, CRs to CS- were not significantly affected by the introduction of $\mathrm{CS}_{3}$ (all contrasts produced $\mathrm{F}$ ratios less than 1.75). By the last trial block (Trials 133-192), however, the 0\% $\mathrm{CS}_{3}$-UCS group showed a significant increase in percent CRs to CS-, $\mathrm{F}(1,30)=6.000, \mathrm{p}<.05$.

\section{Within Group}

When percent $\mathrm{CS}_{3} \cdot \mathrm{UCS}$ pairs was zero, the percent $\mathrm{CRs}$ to $\mathrm{CS}$ - was greater than the percent $\mathrm{CRs}$ to $\mathrm{CS}_{3}$ over both the last two trial blocks, $\mathrm{F}=5.004, \mathrm{p}<.05$. The result was reasonable if one assumes that responding to a tonal CS - would be affected by the response tendency generalized from tonal CS+. No such generalization would be expected from tonal $\mathrm{CS}+$ to $\mathrm{CS}_{3}$ (a red light). It was not clear why the percent CRs over CS- trials increased for the $0 \%$ group and remained stationary for the $20 \%$ group after $\mathrm{CS}_{3}$ was introduced instead of lowered as predicted by excitation-inhibition theory (Gynther, 1957). Peterson and Newman (1970) found responding to CS - to be remarkably stable over 240 differential conditioning trials.

When the percent of $\mathrm{CS}_{3}$-UCS pairings was $20 \%$, a different picture emerged. The percent CRs to CS- and $\mathrm{CS}_{3}$ were approximately equal to each other and approximately at the level of responding shown to be true on $\mathrm{CS}-$ trials prior to introducing $\mathrm{CS}_{3}$ (Trials 49-72). The percent CRs to CS+ dropped initially, as indicated above. On the last trial block, the percent CRs to CS+ returned to its earlier level and the percent CRs to $\mathrm{CS}_{3}$ rose above the level of CRs to CS-. A sign test showed that significantly more Ss (9 of 11 Ss) had a greater number of $\mathrm{CRs}$ to $\mathrm{CS}_{3}$ than to $\mathrm{CS}-$ $(p<.05)$, but a t test did not show significance, $t(22)=$ $1.157, \mathrm{p}>.05$. Thus, aside from the decisively strong linear trend across reinforcement schedule groups for percent $\mathrm{CRs}$ to $\mathrm{CR}_{\mathbf{3}}$, there was little evidence that $\mathrm{Ss}$ in the $20 \%$ group differentiated $\mathrm{CS}_{3}$ and $\mathrm{CS}$ -

For the $60 \% \mathrm{CS}_{3}$-UCS group, the percent CRs to CS+ and to CS- were the least affected by the introduction of $\mathrm{CS}_{3}$. There was a tendency for the number of CRs to CS- on the last two trial blocks to be greater than the number of CRs to CS- during the earlier phase of training (Trials 49-72), but the results of neither the $t$ nor the sign tests were significant.

\section{EXPERIMENT II}

Contrary to Experiment I, the physical character of $\mathrm{CS}_{3}$ in Experiment II was either more similar to CS+ (site of excitation) or to CS- (the site of inhibition). The percent of $\mathrm{CS}_{3}$ pairings was again varied, but here the purpose was to accentuate the extent of generalization from $\mathrm{CS}+$ or $\mathrm{CS}-$ as a function of $\mathrm{CS}_{3}$-UCS association.

\section{Method}

Apparatus, Procedure, and Design

All features were the same except that $\mathrm{CS}_{3}$ was either a 700or a $900-\mathrm{Hz}$ tone. For the design, the additional two-level 
variable of CS similarity was crossed with percent $\mathrm{CS}_{3}-\mathrm{UCS}$ pairings and the counterbalanced assignment of CS+ and CS - to the 600 - and $1,000-\mathrm{Hz}$ tones.

\section{Subjects}

The distinction of "good," "poor," and "nondifferentiating" Ss was also used in Experiment II. The design involved 12 groups in the final partitioning, and Ss were assigned randomly to each partition until five "good" differentiating male Ss were in each. There was a total of 184 male introductory psychology students run, with $16 \mathrm{Ss}$ lost to $\mathrm{E}$ error or apparatus failure. Of the 169 remaining Ss, $79 \%$ showed positive difference scores: $60 / 169$ were "good" and 73/169 "poor" differentiators. Again no significant departures from the expected frequencies were detected ( $p>.10)$, and only the results of "good" Ss were completely analyzed.

\section{Results and Discussion}

The results of the $\mathrm{CS}_{3}$ similar to CS+ groups are summarized in the upper two panels of Fig. 2; the $\mathrm{CS}_{3}$ to CS- group results are presented in the lower two panels.

The adjusted linear trend of $\mathrm{CRs}_{\text {to }} \mathrm{CS}_{3}$ across levels of percent $\mathrm{CS}_{3}$-UCS pairings, while significant, $\mathrm{F}(1,48)=$ $46.57, \mathrm{p}<.001$, appeared to be flatter in Experiment II. The CS similarity effect on the percent CRs to $\mathrm{CS}_{3}$ manifest a significant difference in the adjusted quadratic trends, $F(1,48)=9.52, p<.005$. The difference was apparently due to the smaller increase in the percent $\mathrm{CRs}$ to $\mathrm{CS}_{3}$ from the $0 \%$ group to the $20 \%$ group under the $\mathrm{CS}_{3}$ similar to $\mathrm{CS}+$ condition.

The functions at both levels of CS similarity differed from that observed in Experiment I. When $\mathrm{CS}_{3}$ was more similar to CS--, there were no statistically reliable effects on the percent CRs to CS+ for either the introduction of $\mathrm{CS}_{3}$ trials or the schedule of percent $\mathrm{CS}_{3}$-UCS pairings variable. On the other hand, some rather marked effects were observed when $\mathrm{CS}_{3}$ was more similar to $\mathrm{CS}+$ than to $\mathrm{CS}$-. There was an overall decrease in percent CRs to CS+ after $\mathrm{CS}_{3}$ trials were introduced $F(1,48)=11.19, p<.01$. Moreover, a contrast of linear trends across percent $\mathrm{CS}_{3}$-UCS pairings by trial block was significant, $\mathrm{F}(1,48)=7.14, \mathrm{p}<.025$. The difference in linear trends was due to an increase in percent CRs to CS+ for the $0 \%$ and $20 \%$ groups, but not the $60 \%$ group, $F(1,48)=13.29, p<.01$. Thus, the decrease in the percent CRs to CS+ for the $0 \%$ and $20 \%$ groups was only temporarily affected by $\mathrm{CS}_{3}$ trials.

The introduction of the tonal $\mathrm{CS}_{3}$ trials resulted in an overall increase in the percent CRs to $\mathrm{CS}-, \mathrm{F}(1,48)=$ $10.54, p<.005$. Two interesting effects were found (post hoc) to be influenced by CS similarity. First, when $\mathrm{CS}_{3}$ was more similar to $\mathrm{CS}+$, the increase in percent CRs to CS - was reliable for only the $60 \%$ group, $\mathrm{F}(1,48)=13.33, \mathrm{p}<.05$. Second, for the $\mathrm{CS}_{3}$ similar to CS - condition, the increase in percent CRs to CS- was significant for only the $0 \%$ and $20 \%$ groups, $\mathrm{F}(1,48)=$ $7.21, \mathrm{p}<.05$

Together, these results suggest that generalization between the newly introduced $\mathrm{CS}_{3}$ and either of the established CSs (CS+ or CS--) was greatest when the physical similarity between two CSs was high and when

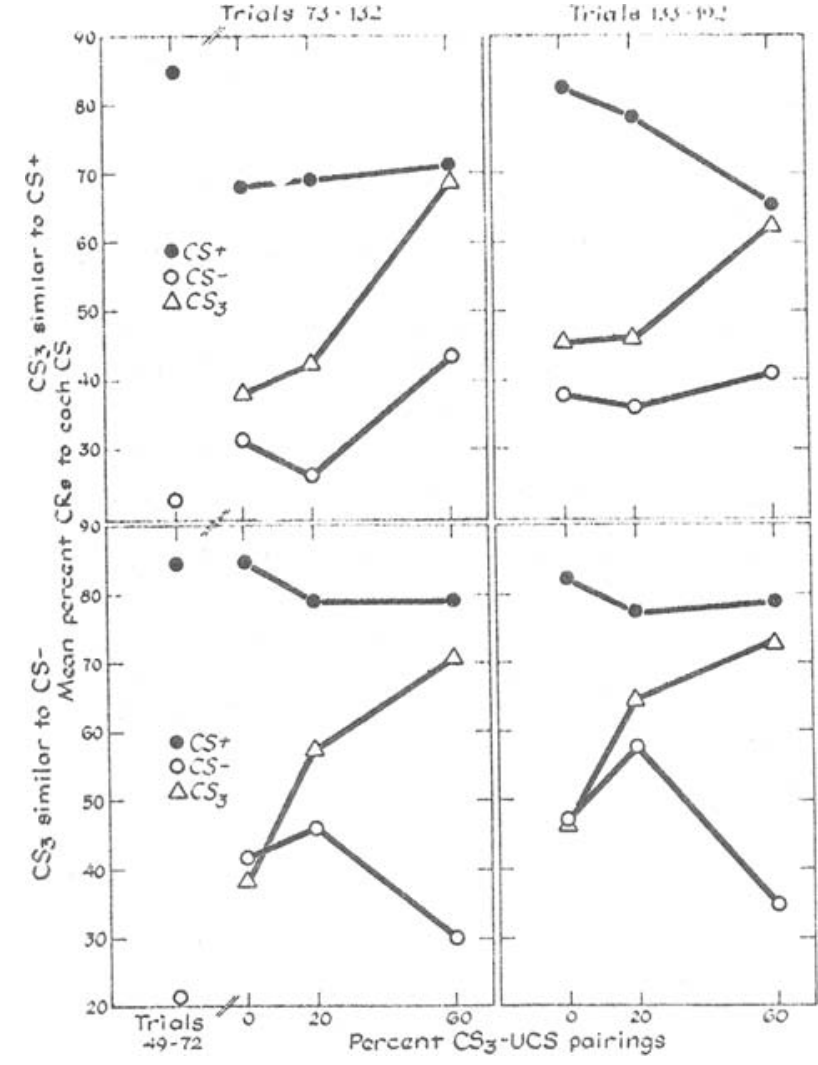

Fig. 2. The mean percent CRs to each CS for Experiment II are given. The effects of $\mathrm{CS}_{3}$ more similar to $\mathrm{CS}+$ condition are presented in the upper two panels. The lower two panels give the results of $\mathrm{CS}_{3}$ more similar to $\mathrm{CS}$ - condition.

the schedule of $\mathrm{CS}_{3}$-UCS pairings was most similar. The interplay between $\mathrm{CS}_{3}$ and $\mathrm{CS}+$ was greatest when $\mathrm{CS}_{3}$ was more similar to $\mathrm{CS}+$ than $\mathrm{CS}$ - and when the percent $\mathrm{CS}_{3}$-UCS pairing was $60 \%$. Likewise, the interplay between $\mathrm{CS}_{3}$ and $\mathrm{CS}$ - was greatest when $\mathrm{CS}_{3}$ was more similar to $\mathrm{CS}-$ than $\mathrm{CS}+$ and when the percent $\mathrm{CS}_{3}$-UCS pairings was either $0 \%$ or $20 \%$.

The fact that the strongest generalization effects were observed between two Ss when both CS similarity and similarity of reinforcement schedules were high was anticipated. On the other hand, the nature of the effects was not predictable from excitation-inhibition theory. The inconsistencies with theory can best be seen by considering the two extreme cases of high stimulus and schedule similarity. Theory predicts that generalized effects of reinforcement would be greatest when $\mathrm{CS}_{3}$ and $\mathrm{CS}+$ were similar and the $\mathrm{CS}_{3}$-UCS schedule was $60 \%$. The surprising result was the marked decrease in the percent CRs on CS+ trials. The decrease in percent CRs to CS+ was such that there were no differences in percent CRs on CS+ and $\mathrm{CS}_{3}$ trials. Moreover, the percent $\mathrm{CRs}$ on $\mathrm{CS}_{3}$ trials was no higher in this group than in any other group. It is difficult to conceive that the $40 \%$ nonreinforced $\mathrm{CS}_{3}$ trials in the $60 \%$ group were more effective in generalizing inhibitory effects than they were in the $0 \%$ or $20 \%$ groups. For the $0 \%$ and $20 \%$ 
groups ( $\mathrm{CS}_{3}$ and $\mathrm{CS}+$ similar), an initial decrease in percent CRs to CS+ was observed, but the decrease did not persist into the second block of 60 trials as was observed in the $60 \%$ group.

At the other extreme was the $0 \%$ group, for which $\mathrm{CS}_{3}$ was more similar to $\mathrm{CS}$ - than $\mathrm{CS}+$, and the inhibitory effects should have been optimal. The introduction of the nonreinforced $\mathrm{CS}_{3}$ trials resulted in a reliable increase in the percent CRs to CS- equal to the level observed on $\mathrm{CS}_{3}$ trials. Furthermore, the percent $\mathrm{CRs}$ on $\mathrm{CS}+$ trials maintained at the pre-CS level instead of decreasing. A decrease was anticipated because of the increased potential for generalized inhibition from both $\mathrm{CS}_{3}$ and $\mathrm{CS}-$. The same set of results was observed in the $20 \%$ group with $\mathrm{CS}_{3}$ similar to CS-. Specifically the percent CRs to $\mathrm{CS}_{3}$ and to CSfor the $20 \%$ group were relatively higher under the $\mathrm{CS}_{3}$ similar to CS- condition than under the reversed CS similarity condition. Furthermore, the percent CRs on $\mathrm{CS}_{3}$ trials were lower for the $60 \%$ group under the $\mathrm{CS}_{3}$ similar to CS+ condition. As such, the results were opposed to an excitation-inhibition interpretation. Recently, Allan \& Branum (1971) suggested that gradients of generalized excitation and inhibition would be flatter in situations involving partial reinforcement. Even the extension of excitation-inhibition theory proposed by Allan and Branum fails to justify the inconsistencies found here.

The present experimental results showed that excitation-inhibition theory did not provide a satisfactory explanation for a two-phase conditioning procedure. Since the formal analysis used the data of only those Ss who were clearly differentiating at the end of the first conditioning, one is tempted to postulate the additional effects of awareness (Perry, Grant, \& Schwartz, 1971) or the information signaling properties of the various CSs (Grant, 1968). While the first training phase served to establish the differential conditioning process, the introduction of $\mathrm{CS}_{3}$ in the second phase may have had differential cuing effects of the sort described by Grant (1972).

\section{REFERENCES}

Allan, C. K., \& Branum, A. R. Differential eyelid conditioning as a function of the probability of reinforcement of the conditioned stimuli. Psychonomic Science, 1971, 22, 251-252.

Grant, D. A. Adding communication to the signaling properties of the CS in classical conditioning. The Journal of General Psychology, 1968, 79, 147-175.

Grant, D. A. A preliminary model for processing information in classical differential conditioning. In $A$. H. Black and W. F Prokasy (Eds.), Classical conditioning II. New York: Appleton-Century-Crofts, 1972.

Gynther, M. D. Differential ey elid conditioning as a function of stimulus similarity and strength of response to the CS. Journal of Experimental Psychology, 1957, 53, 408-416.

Perry, L. C. Grant, D. A. \& S chwartz, M. Differential eyelid conditioning with grammaticality of a noun phrase as the discriminandum. Paper presented at the meeting of the Psychonomic Society, St. Louis, November 1971.

Peterson, G. B., \& Newman, F. L. Differential human eyelid conditioning as a function of the probability of reinforcement and CS similarity. Journal of Experimental Psychology, 1970, 85, 318-320.

\section{NOTES}

1. Female ss did not volunteer in sufficient numbers to provide a proportionately balanced design.

2. All analyses were performed on the arcsin transforms of the percent CRs. All trend analyses involved coefficients adjusted for the unequal intervals across the percent $\mathrm{CS}_{3}$-UCS pairing variable $(0 \%, 20 \%$, and $60 \%)$.

(Received for publication April 7, 1973.) 